\title{
Validación del índice nutricional en preadolescentes mexicanos con el método de sensibilidad y especificidad*
}

Teresita de Jesús Saucedo-Molina, Lic. en N utr., M. en Psic. Educ., ${ }^{(1)}$

Gilda Gómez-Peresmitré, Lic. en Psic., Dr. en Psic.(2)

\section{Saucedo-Molina TJ, Gómez-Peresmitré G. Validación del índice nutricional en preadolescentes mexicanos con el método de sensibilidad y especificidad. Salud Publica Mex 1998;40:392-397.}

\begin{abstract}
Resumen
Objetivo. D eterminar la validez diagnóstica del índice nutricional (IN) en una muestra de preadolescentes mexicanos. Material y métodos Se trabajó con un grupo de 256 escolares de la Ciudad de México, de entre 10 y 12 años de edad, de ambos sexos. Se utilizó el método de sensibilidad y especificidad para establecer la validez diagnóstica del IN . Resultados Los puntos de corte convencionales para el IN tienen buena sensibilidad y especificidad para el diagnóstico de bajo peso, normalidad y obesidad, no así para sobrepeso. Estos valores, así como los de la potencia de predicción, se optimizaron en todas las categorías cuando se utilizaron puntos de corte normalizados. Conclusiones. En el diagnóstico del estado de nutrición de los preadolescentes, los nuevos puntos de corte del IN resultan más confiables que los tradicionales.
\end{abstract}

Palabras clave:evaluación nutricional/validez; antropometría; preadolescentes; sensibilidad y especificidad; México
Saucedo-Molina TJ, Gómez-Peresmitré G. Validation of the nutritional index in Mexican preadolescents with the sensitivity and specificity method.

Salud Publica Mex 1998;40:392-397.

\begin{abstract}
A bstract
Objetive To determine the diagnostic validity of the nutritional index (N I) in a sample of Mexican preadolescents. Material and methods A total of 256 preadolescents, between 10 and 12 years old, male and female, students from Mexico City, were used to establish the diagnostic validity of $\mathrm{NI}$ using the sensitivity and specificity method. Results. The findings show that the conventional $\mathrm{N}$ I cut-off points showed good sensitivity and specificity for the diagnosis of low weight, normality and obesity but not for overweight. W hen the cut-off points of NI were normalized, the sensitivity, specificity and prediction potency values were more suitable in all categories. Conclusions W hen working with preadolescents, it is better to use the new cut-off points of $\mathrm{NI}$, to obtain more reliable diagnosis.
\end{abstract}

Key words: nutrition assessment/validity; anthropometry; preadolescents; sensitivity and specificity; Mexico
L a evaluación antropométrica consiste en la medición de las dimensiones y la composición global del cuerpo humano, las que indudablemente se ven afectadas por la nutrición durante el ciclo de vida. Los indicadores antropométricos miden, por un lado, el crecimiento físico del niño y del adolescente, así como las dimensiones físicas del adulto, a partir de la determinación de la masa corporal total (peso y talla) y, por el otro, la composición corporal, es decir, la masa grasa y la masa libre de grasa (pliegues y circunferencias). ${ }^{1}$

Los indicadores antropométricos son considerados como un reflejo aproximado del estado nutricio, y las mediciones que se usan con más frecuencia son el

* Esta investigación forma parte del proyecto IN 304496, financiado por PAPIIT-96 UNAM.

(1) Escuela de Dietética y N utrición. Instituto de Seguridad y Servicios Sociales de los Trabajadores del Estado, México.

(2) Facultad de Psicología. Universidad N acional Autónoma de México, México.

Fecha de recibido: 21 de octubre de 1997 - Fecha de aprobado: 31 de julio de 1998 Solicitud de sobretiros: Mtra. Teresita de Jesús Saucedo Molina. Rinc. de los Juegos Edif. Lotería 203, colonia Pedregal de Carrasco, 04700 México, D.F., México. 
peso corporal y la altura, así como los perímetros craneal y del brazo, y los pliegues cutáneos. Estas técnicas se utilizan debido a su fácil aplicación, bajo costo y reproducibilidad en diferentes momentos y con distintas personas. Asimismo, permiten valorar la masa corporal y su composición en diversas etapas de la vida, tanto en la salud como en la enfermedad. ${ }^{2}$

Dos son las mediciones que, dadas la calidad de información que aportan como indicadores y la factibilidad de su levantamiento, pueden ser consideradas como las más adecuadas: el peso y la talla, mismos que, referidos a la edad y el sexo, permiten establecer las adecuaciones respecto a la norma de peso para la talla (nutrición actual), talla para la edad (nutrición histórica) y peso para la edad (nutrición actual e histórica). ${ }^{3}$

Otros indicadores que han demostrado ser de gran utilidad para valorar el estado de nutrición son: el perímetro cefálico, el perímetro del brazo y el grosor de los pliegues cutáneos. Para hacerlos más sensibles e independientes de la edad, algunos de estos indicadores se han combinado entre sí, lo que a su vez ha dado origen a diversos índices que facilitan su interpretación y permiten hacer una clasificación más precisa sobre las alteraciones de la nutrición, tanto por exceso como por deficiencia. Entre los más utilizados en salud pública, están el índice nutricional (IN), el perímetro brazo/perímetro cefálico y el índice de masa corporal (IMC). ${ }^{4}$

Durante los últimos cinco años, en México se han desarrollado múltiples programas para recabar datos antropométricos que permitan evaluar el estado nutricional en casi todos los estados de la República. Sin embargo, cabe mencionar que prácticamente todas esas investigaciones se llevaron a cabo en menores de seis años o en mujeres embarazadas, ${ }^{5}$ por considerárseles como grupos vulnerables. Las características de esos estudios hacen evidente la necesidad de contar con guías sobre el uso apropiado y la interpretación de indicadores e índices antropométricos válidos a lo largo de todo el ciclo de vida. ${ }^{6}$

Uno de los periodos más significativos en el crecimiento y la maduración del ser humano es la adolescencia; se trata de una transición y un enlace entre la infancia y la condición adulta. La proximidad de la adolescencia a una madurez biológica y a la adultez significa la última oportunidad para prevenir problemas de salud en la vida adulta. Esta etapa se caracteriza por un aumento brusco y considerable de todas las dimensiones musculares y esqueléticas del cuerpo, llamada brote de crecimiento adolescente y que se refleja en los valores de talla, peso y superficie corporal, así como en los diámetros, perímetros y relación de segmentos.
La adolescencia es un proceso evolutivo cambiante común a todo ser humano, que se anuncia en la etapa puberal llamada también adolescencia temprana o preadolescencia; es decir, la adolescencia comienza con la aparición de los signos sexuales secundarios (entre los 10 y los 12 años), continúa con la adolescencia media en la que los cambios puberales ya se encuentran establecidos (de los 13 a los 15 años) y termina en la adolescencia final, que puede ir desde los 16 hasta los 18 a 20 años de edad, de acuerdo con el momento en el cual el adolescente se inserta en el mundo y la vida social del adulto. ${ }^{7}$

A pesar de que la adolescencia es claramente un periodo de suma importancia en el desarrollo humano, aún no se le ha dado la debida atención desde el punto de vista antropométrico, de tal manera que no se han establecido aplicaciones e interpretaciones antropométricas directamente vinculadas con los riesgos para la salud. Los rápidos cambios en el crecimiento somático durante la adolescencia, los problemas de la distribución en cuanto a la variación en la maduración y las dificultades que representa la separación de las variaciones normales de aquellas asociadas con riesgos de salud, han sido, desde siempre, razones que han desalentado el desarrollo de conocimientos más confiables acerca de la antropometría de la adolescencia. ${ }^{8}$

Al llevar a cabo la revisión bibliográfica necesaria para el desarrollo de la presente investigación, se encontraron algunos estudios antropométricos realizados en preadolescentes y adolescentes. ${ }^{9-11}$ No obstante, en ninguno se validan índices para la clasificación nutricional de este tipo de población, con excepción de los realizados por Gómez Peresmitré y colaboradores, en los cuales se determinó la validez diagnóstica de tres criterios de IMC en preadolescentes y adolescentes mexicanos; se encontró que los niveles de sensibilidad y especificidad para el grupo de preadolescentes resultaron poco confiables para el diagnóstico de bajo peso, sobrepeso y obesidad, de tal manera que no se recomienda su empleo en este tipo de población. ${ }^{12,13}$ Por este motivo, el objetivo fundamental de este estudio fue determinar la validez diagnóstica de un índice antropométrico utilizado en salud, en una población de riesgo poco estudiada como son los preadolescentes (adolescencia temprana); cabe aclarar que se consideró como tales a aquellos sujetos de ambos sexos que se encuentran entre los $10 \mathrm{y}$ los 12 años de edad.

Se seleccionó el IN para someterlo a validación por medio del método de sensibilidad y especificidad. Una de las razones para seleccionarlo fue que sus parámetros de comparación son el peso y la talla. Cabe señalar que el peso, la talla y la edad son las mediciones básicas que debe registrar un sistema de vigilancia 
epidemiológica de la nutrición, ya que los índices construidos a partir de estos datos ofrecen una amplia gama de posibilidades de análisis que revelan las características de la nutrición actual e histórica del conjunto de individuos bajo vigilancia, su riesgo de desnutrición $u$ obesidad, su evaluación a lo largo del tiempo y el impacto de los programas de alimentación y nutrición aplicados. ${ }^{14}$

Como ya se mencionó, el IN se basa en la comparación de la relación simple del peso y la talla del sujeto, con la relación del peso y la talla correspondientes al percentil 50 (mediana), referidos en las tablas del National Center of Health Statistics-Organización Mundial de la Salud (NCHS-OMS), considerando el sexo y la edad. Su fórmula es la siguiente: ${ }^{4}$

$$
\text { Indice nutricional= }
$$

$\frac{\text { Peso actual en } \mathrm{kg} / \text { talla actual en } \mathrm{m}}{\text { Peso en } \mathrm{kg}(\text { percentil 50)/ talla en m (percentil 50) }} \times 100$

El valor de este índice permite diferenciar cuatro situaciones:

*Inferior a $90 \%$ = bajo peso

*Entre $90-110 \%=$ peso normal

*Entre $110-120 \%=$ sobrepeso

*Mayor a $120 \%=$ obesidad

La validez diagnóstica es un método para precisar la confiabilidad de una prueba diseñada para detectar un determinado síndrome o característica. Los criterios para evaluar la calidad de medición de una prueba de diagnóstico se basan en los estudios sobre la sensibilidad, la especificidad y la potencia de predicción de dicha prueba. ${ }^{15}$

Se define como sensibilidad a la capacidad de la prueba para diagnosticar como positivos a quienes en realidad tienen la característica en estudio, es decir, el porcentaje de aciertos o "positivos verdaderos", identificados como tales por la prueba en evaluación respecto a un estándar de oro. Por otro lado, se entiende por especificidad a la capacidad de la prueba para diagnosticar como negativos a quienes en realidad no tienen la característica en estudio, es decir, aquellos sujetos que son "negativos verdaderos" (cuadro I).

Para complementar una prueba de validez diagnóstica, se recomienda determinar su potencia o valor predictivo; ${ }^{16}$ este último es la probabilidad de que un sujeto tenga la característica en estudio cuando la prueba diagnostica positivo. Una prueba con valor predictivo positivo (VPP) de $90 \%$ indica que de cada 100 sujetos cuya prueba resulta positiva, 90 tienen la carac-

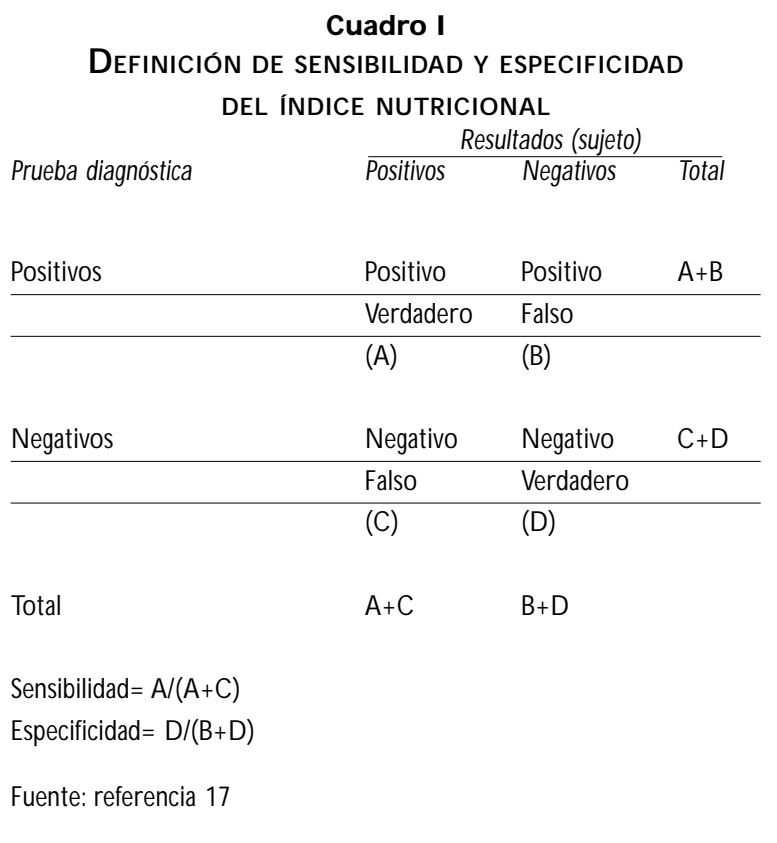

terística buscada, y se detecta un $10 \%$ de positivos falsos $(\mathrm{PF})$. Una prueba con valor predictivo negativo (VPN) de 95\% indicaría que de 100 sujetos con un resultado negativo, 95 carecen del síndrome, y el 5\% restante sería de negativos falsos (NF). El valor predictivo se encuentra íntimamente vinculado a los valores de sensibilidad y de especificidad de la prueba (cuadro II).

\section{Material y métodos}

Durante el segundo semestre de 1996, se llevó a cabo un estudio transversal de campo, donde de una muestra de $n=1383$ escolares preadolescentes de 10 a 12 años $(X=10.98)$ de ambos sexos (mujeres $49 \%$, hombres $51 \%$ ), se extrajo una submuestra por selección sistemática aleatoria de $18 \%(\mathrm{n}=256)$, de la que se obtuvieron mediciones antropométricas, tales como peso, talla y circunferencia de brazo. Dichas mediciones se recabaron de acuerdo con las técnicas internacionalmente recomendadas, mediante la participación de cuatro encuestadores estandarizados con antelación. ${ }^{19}$

Se utilizaron las referencias de peso y talla provenientes de las tablas NCHS-OMS, ${ }^{20}$ ya que:

A pesar de ser una norma para la población estadounidense, se trata de una muestra multiétnica, multiestratificada, paramétrica y controlada en cuanto a normalidad de los sujetos de estudio. Además, en múltiples reportes ha quedado establecido que el crecimiento lineal y ponderal de la población bien nutrida nativa de los países tercermundistas es muy similar a la norma NCHS-OMS. ${ }^{5}$ 


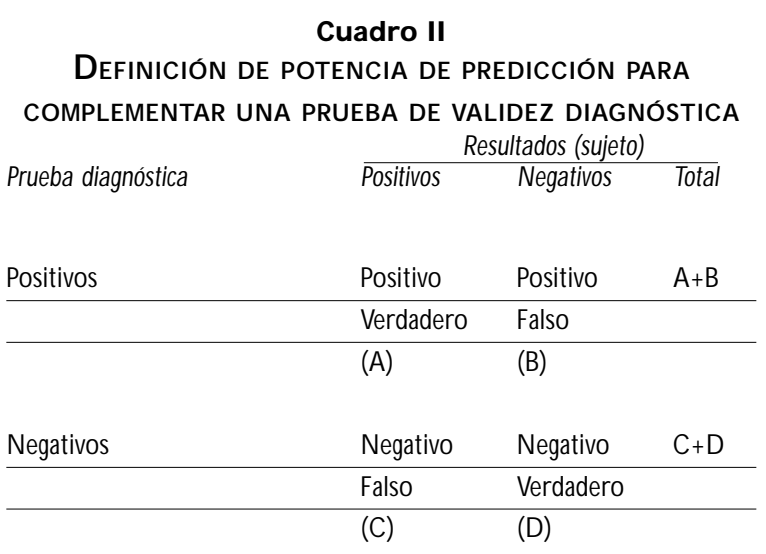

Valor predictivo positivo, $V P P=A /(A+B)$

Valor predictivo negativo, $V P N=D /(C+D)$

Fuente: referencia 18

Por otra parte, se utilizó el método de validez diagnóstica haciendo la comparación entre los puntos de corte del IN contra la cincunferencia de brazo, considerado como estándar de oro porque es un indicador ampliamente utilizado y validado internacionalmente, ${ }^{16,21-23}$ al respecto se ha detectado que la diferencia entre sexos en grupos de púberes y adolescentes es poco significativa, ${ }_{11}^{11}$ además de que, tanto el peso en relación con la talla, como la circunferencia de brazo, son indicadores de masa corporal.

Los puntos de corte para la circunferencia de brazo fueron:

*Inferior al percentil 10= malnutrición o bajo peso

${ }^{*}$ Entre los percentiles 10 y $75=$ normalidad

${ }^{*}$ Entre los percentiles 75 y $90=$ sobrepeso

${ }^{*}$ Mayor al percentil 90= obesidad ${ }^{24}$

En forma adicional se decidió hacer, de la misma submuestra, una distribución normalizada ajustando la media (trim mean), ${ }_{1}^{25}$ con la finalidad de determinar cómo se modificarían tanto la sensibilidad y la especificidad, como la potencia de predicción del IN al utilizar nuevos puntos de corte. La media se ajustó al 5\%, esto es, se eliminaron $5 \%$ de los valores extremos más altos y $5 \%$ de los más bajos. Posteriormente, con base en la distribución resultante -y que representaba a la submuestra de 256 preadolescentes de 10 a 12 años de edad de ambos sexos- se efectuó una división en deciles, de tal manera que los valores del lado izquierdo de la curva que se encontraron por debajo de $10 \%$ correspondieron a la categoría peso muy bajo, y los va- lores del lado derecho que cayeron por arriba de $90 \%$, a la obesidad. Del $80 \%$ restante, $40 \%$, representado por el valor más alto, correspondió a la normalidad, y los valores extremos de $20 \%$ cada uno, fueron para las categorías de peso bajo y sobrepeso, respectivamente.

Los puntos de corte resultantes de este paso fueron:

${ }^{*}$ Entre 77.51 y $83.5 \%=$ bajo peso

*Entre 83.51 y $119.5 \%$ = peso normal

*Entre 119.51 y $136.5 \%=$ sobrepeso

${ }^{*}$ Mayor a $136.5 \%=$ obesidad

\section{Resultados}

Los hallazgos al analizar la submuestra indican que, para el diagnóstico de bajo peso (por debajo de 90\%), el IN convencional presenta un buen equilibrio entre sensibilidad (93\%) y especificidad (89\%), ya que estos porcentajes indican que de 100 sujetos de bajo peso, se identifica a $93 \%$ de los que en realidad lo tienen $y$, además, discrimina al $89 \%$ como sujetos sin bajo peso cuando verdaderamente no lo tienen. Sin embargo, cabe destacar que la potencia de predicción, misma que alcanzó valores de $\mathrm{VPP}=48 \%$ y $\mathrm{VPN}=98 \%$, mejoró cuando se determinó la validez del IN normalizado (entre 77.51 y $83.5 \%$ ), cuya sensibilidad y especificidad fueron de 67 y 97\%, respectivamente; de este modo, se registraron valores de $\mathrm{VPP}=75 \%$ (probabilidad de 0.75 ) y VPN= 95\% (probabilidad de 0.95).

En cuanto a la determinación del peso normal (entre 90 y 110\%) del IN convencional, los valores de sensibilidad y especificidad (65 y 92\%, respectivamente), presentaron un equilibrio aceptable, aunque éste se incrementó cuando se validó el punto de corte del IN normalizado (entre 83.51 y $119.5 \%$ ), cuya sensibilidad y especificidad resultó ser más confiable $(93$ y $75 \%$, respectivamente), pues dichos porcentajes indican que, de cada 100 sujetos diagnosticados como normales, 93\% efectivamente lo son y, por el contrario, que de cada 100 sujetos detectados como no normales, $75 \%$ efectivamente corresponden a dicha categoría. Además, también se mejoró el equilibrio de la potencia de predicción, pues de valores de VPP= 91 y VPN= 67 en el IN convencional, se registraron valores de $\mathrm{VPP}=81 \mathrm{y}$ $\mathrm{VPN}=90$ para el IN normalizado.

En cuanto a los valores obtenidos para sensibilidad y especificidad respecto al diagnóstico de sobrepeso, se aprecia que el punto de corte correspondiente al IN convencional (entre 110 y 120\%) tiene valores sumamente desequilibrados (26 y $90 \%$, respectivamente), además de registrar un valor de predicción positivo sumamente bajo $(\mathrm{VPP}=32 \%)$. Cuando se validó el punto de corte del IN normalizado (entre 119.51 y $136.5 \%$ ), 
los porcentajes de sensibilidad y especificidad tendieron a equilibrarse (52 y 96\%, respectivamente), así como la potencia de predicción, pues el VPP, que con el IN convencional fue de $36 \%$, subió hasta $69 \%$, de tal manera que si se emplea este punto de corte normalizado se tendrá la certeza de que, de cada 100 sujetos, por lo menos $52 \%$ serán diagnosticados correctamente con sobrepeso, y $96 \%$, como sujetos sin sobrepeso. Por otra parte, existe una probabilidad de 0.76 de que, mediante este punto de corte, un preadolescente de la muestra original $(n=1383)$ que haya sido diagnosticado con sobrepeso verdaderamente lo sea, y una probabilidad de 0.90 de que no tenga sobrepeso si la prueba así lo diagnostica.

Finalmente, en cuanto a la validez diagnóstica para obesidad, se aprecian excelentes valores de sensibilidad y especificidad para ambos puntos de corte, pues el IN convencional (mayor a 120\%) notificó 93 y 85\%, respectivamente, contra 71 y $97 \%$ del IN normalizado (mayor a $136.51 \%$ ). Sin embargo, es evidente que la potencia de predicción mejora al normalizar el IN, pues el VPP de 53\% que alcanzó el IN convencional puede incrementarse hasta $83 \%$; es decir, con el punto de corte normalizado existe la probabilidad de 0.83 de que un sujeto de la muestra que haya sido identificado como obeso verdaderamente lo sea, así como la probabilidad de 0.95 de que un preadolescente que haya sido detectado como no obeso en realidad no lo sea (cuadro III).

\section{Discusión}

Tres razones fundamentales son las que dieron origen a la presente investigación: en primer lugar, la mayoría de los programas para recabar datos antropométricos realizados en México se orientan principalmente a los menores de seis años de edad y a las mujeres embarazadas. ${ }^{5}$ En segundo lugar, existe gran polémica respecto a cuáles son los mejores índices para detectar el estado nutricio durante la adolescencia, por lo que profundizar en el estudio de indicadores antropométricos para optimar su utilidad en la evaluación nutricional en esta etapa resulta de vital interés. Por último, es poca la experiencia que se tiene en el uso de indicadores confiables para la evaluación del estado nutricional durante la adolescencia.

Por este motivo, el presente estudio abordó una población sociodemográfica relevante para la salud pública, como lo es la de adolescentes, específicamente la de preadolescentes. Asimismo, este trabajo representa uno de los pocos intentos que se han hecho ${ }^{12,13}$ para detectar la validez diagnóstica de índices antropométricos recomendados en el área de la salud ${ }^{4}$ para este tipo de población, utilizados para estimar el estado de

\section{Cuadro III \\ SENSIBILIDAD, ESPECIFICIDAD Y POTENCIA \\ DE PREDICCIÓN PARA LA CLASIFICACIÓN DEL ESTADO NUTRICIO EN PREADOLESCENTES}

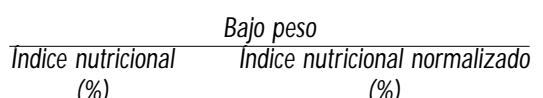

$(\%)$

$(\%)$

\begin{tabular}{lcc} 
Puntos de corte & Inferior a 90 & Entre 77.51 y 83.5 \\
\hline Sensibilidad & 93 & 67 \\
\hline Especificidad & 89 & 97 \\
\hline VPP & 48 & 75 \\
\hline VPN & 98 & 95
\end{tabular}

\begin{tabular}{lcc} 
& \multicolumn{3}{c}{ Peso normal } \\
\cline { 2 - 3 } Puntos de corte & Entre 90 y 110 & Entre 83.51 y 119.5 \\
\hline Sensibilidad & 65 & 93 \\
\hline Especificidad & 92 & 75 \\
\hline VPP & 91 & 81 \\
\hline VPN & 67 & 90
\end{tabular}

\begin{tabular}{lcc} 
& \multicolumn{2}{c}{ Sobrepeso } \\
\cline { 2 - 3 } Puntos de corte & Entre 110 y 120 & Entre 119.51 y 136.5 \\
\hline Sensibilidad & 26 & 52 \\
\hline Especificidad & 90 & 96 \\
\hline VPP & 32 & 69 \\
\hline VPN & 85 & 90
\end{tabular}

\begin{tabular}{lcc} 
& \multicolumn{2}{c}{ Obesidad } \\
\cline { 2 - 3 } Puntos de corte & Mayor a 120 & Mayor a 136.5 \\
\hline Sensibilidad & 93 & 71 \\
\hline Especificidad & 85 & 97 \\
\hline VPP & 53 & 83 \\
\hline VPN & 98 & 95
\end{tabular}

VPP: valor predictivo positivo o probabilidad de que un sujeto diagnosticado positivo, en realidad tenga la característica buscada

VPN : valor predictivo negativo o probabilidad de que un sujeto diagnosticado como negativo, en realidad no tenga la característica buscada

nutrición de un individuo con base en la medición y composición corporal. ${ }^{1}$

De acuerdo con los resultados de sensibilidad y especificidad y puesto que diversos autores ${ }^{5,9,14}$ consideran que el peso y la talla son las mediciones básicas que debe registrar un sistema de vigilancia epidemiológica de la nutrición, se concluye que en los preadolescentes mexicanos los puntos de corte del IN convencional tienen una validez diagnóstica aceptable, aunque no en todas sus categorías, y que la misma se puede mejorar utilizando puntos de corte no convencionales. Por ejemplo, cuando se tiene un punto de corte de entre 110 y 120\% recomendado para la clasi- 
ficación de sobrepeso, los valores de sensibilidad y especificidad resultan de 26 y $90 \%$ respectivamente, y al modificar los puntos de corte (entre 119.51 y $136.5 \%$ ) por medio de la técnica trimmed mean, ${ }^{25}$ esos valores tienden a equilibrarse, indicando una sensibilidad de $52 \%$ y una especificidad de $96 \%$. Lo anterior se fundamenta en el hecho de que precisamente en esta población se inicia el brote de crecimiento característico de la etapa adolescente, mismo que implica cambios psicológicos, fisiológicos y anatómicos que tienen fuerte impacto en las necesidades nutricionales del joven, lo que se refleja directamente en su estado nutricio. ${ }^{26}$

De acuerdo con la OMS, los indicadores e índices antropométricos son vitales en la evaluación del estado nutricio de las poblaciones, y su uso se enfoca, sobre todo, a la identificación de prevalencia, incidencia, y severidad de la desnutrición y la obesidad. Por ello, es fundamental contar con criterios válidos para cada tipo de población, ${ }^{6,8}$ pues no sólo son útiles para detectar grupos de riesgo, sino que permiten establecer programas de vigilancia nutricional y de prevención primaria, objetivo final de esta investigación.

Es necesario continuar con este tipo de investigaciones utilizando metodologías más sensibles que permitan obtener puntos de corte que puedan ser generalizados en poblaciones similares. Un ejemplo de dichas metodologías son las llamadas curvas ROC (Receiver Operating Characteristics). ${ }^{27,28}$ De igual manera, es claro que el procedimiento de normalización empleado en este estudio requiere de afinación, lo que se convierte en un objetivo prioritario de estudios posteriores.

\section{Referencias}

1. Casanueva $E$, Kaufer-H orwitz M, Pérez-Lizaur AB,Arroyo P. N utriología médica. México, D.F.: Médica Panamericana, 1995:72-84, 470-492.

2. Jelliffe $E P$, Jelliffe $D B$. Community nutritional assessment with special reference to less technically developed countries. $N$ ueva York: 0 xford University Press, 1989.

3. Briend $A$, H asen Zkh,Azis KMA, H oque BA, Henry FJ. Measuring change in nutritional status: A comparision of different anthropometric indexes and the sample sizes required. Eur J C lin N utr 1989:43:769-778.

4. Hernández-Rodríguez M.A limentación infantil. Madrid: Díaz de Santos, 1993;11-23.

5. Chávez A.Vigilancia epidemiológica de la nutrición. México, D.F.: Instituto N acional de la N utrición Salvador Zubirán, 1990.

6 . W orld Health 0 rganization. Used and interpretation of anthropometric indicators of nutritional status. Bull W orld Health O rgan 1986;64(6): 929-941.

7. Martínez y Martínez R. La salud del niño y del adolescente. Federación de Pediatría Centro-O ccidente de México. 3a. edición. México, D.F.: JGH Masson-Salvat, 1996: 1119-1131.
8. 0 nis $M$, Habicht JP. Anthropometric reference data for international use: Recommendations from aW orld Health 0 rganization Expert Committee. Am J Clin N utr 1996;64:650-658.

9. Ramos-Galván R. Significado y empleo de las referencias somatométricas de peso y talla en la práctica pediátrica y epidemiológica. Bol Med Hosp Infant Mex 1992;49(6):321-334.

10. Ramos-Rodríguez RM. Crecimiento y proporcionalidad corporal en adolescentes mexicanas. México, D.F.: Universidad $\mathrm{N}$ acional Autónoma de México, 1986.

11. Faulhaber J. Crecimiento: somatometría de la adolescencia. México, D.F.: Universidad N acional Autónoma de México, 1989.

12. Saucedo-MolinaTJ, Gómez-Peresmitré G Validez diagnóstica del índice de masa corporal en adolescentes mexicanos. Acta Pediatr Mex 1997; 18(1):19-27.

13. Gómez-Peresmitré G, Saucedo-MolinaTJ.Validez diagnóstica del índice de masa corporal en una muestra de escolares preadolescentes y adolescentes mexicanos. Acta Pediatr Mex 1997;18(3):103-110.

14.G onzález-RichmondA. Estudio comparativo de diferentes índices antropométricos y sistemas de clasificación del estado nutricional. México, D.F.: Instituto N acional de la N utrición Salvador Zubirán, 1982; L-47.

15. Kelsey JL, Thompson DW. Methods in observational epidemiology. N ueva York: O xford University Press, 1986:285-289.

16. Calero-Rey J. Método epidemiológico y salud de la comunidad. México, D.F.Interamericana/McG raw-Hill, 1989:173-189.

17. González-Richmond A, N aranjo-BandaA. Estudio comparativo de diferentes índices antropométricos del estado nutricional. Bol Med Hosp Infant Mex 1984;41(11):594-604.

18. Moreno-Altamirano L, Cano-Valle F, García-Romero. Epidemiología clínica. México, D.F.: Interamericana/McG raw-Hill, 1994:143-168.

19. Lohman GT, Roche AF, Martorell R. Anthropometric standardization reference manual. Illinois:A Aridged Edition, Human Kinetics Books, 1988. 20. 0 rganización Mundial de la Salud. Medición del cambio del estado nutricional: directrices para evaluar el efecto nutricional de programas de alimentación suplementaria destinados a grupos vulnerables. Ginebra: OMS, 1983:70-89.

21. Jelliffe EPF, Jelliffe $D B$.The arm circunference as a public health index of protein-caloric malnutrition of early childhood. J Trop Pediatr 1969;15: 179-188.

22. Trowbridge FL, Staehling N. Sensitivity and specificity of arm circunference indicators in identifying malnurished children. Am J Clin Nutr 1980;33:687-696.

23. Marín-Flores A, González-Perales C, Alonso-Ramírez E, Beltrán-Villa M. Circunferencia de brazo como indicador de riesgo de desnutrición en preescolares. Salud Publica Mex 1993;35(6):667-672.

24. Frisancho AR. N ew norms of upper limb fat and muscle areas for assessment of nutritional status. Am J C lin N utr 1981;34:2540.

25. N orusis JM. SPSS for windows base system users guide release 5.0 . Michigan:SPSS Inc, 1992.

26. D wyner J. Nutrition and the adolescent. Textbook of pediatric nutrition. N ueva York: Lewiter Suskind Raven Press, 1993.

27. Cho MJ, Moscicki EK, Narrow W E, Rae DS, Locke BZ, Rugev DA. Concordance between two measures of depression in the Hispanic health and nutrition examination survey. Soc Psychiatry Psychiatr Epidemiol 1993;28(4):156-163.

28. Grover SA, Palmer CS, Coupal L. Serum lipid screening to identify high risk individuals for coronary death.The results of the Lipid Research Clinics prevalence cohort. Arch Intern Med 1994;154(6):679-684. 